\title{
CLASSIFICAÇÃO DE VULNERABILIDADE DE ÁREAS À ROUBOS
}

\author{
Amanda Gadelha Ferreira Rosa \\ Universidade Federal de Pernambuco \\ amandagadelharosa@hotmail.com \\ Caroline Maria de Miranda Mota \\ Universidade Federal de Pernambuco \\ carol3m@gmail.com \\ Ciro Figueiredo \\ Universidade Federal de Pernambuco \\ figueiredo@yahoo.com.br
}

\begin{abstract}
RESUMO
A aplicação do modelo de decisão no contexto da segurança pública tornase interessante, pois possui a capacidade de fundamentar as escolhas do decisor segundo alguns pressupostos matemáticos. E, dado as restrições orçamentárias e de pessoal, as ações de alocação de recursos devem ser aquelas que satisfaçam as limitações inerentes no contexto analisado, ao mesmo tempo em que apresentam boas soluções. Sob essa perspectiva, a classificação dos níveis de vulnerabilidade dos locais devem ser consideradas na atribuição das ações de mitigação ao crime. Desta forma, aplicou-se o modelo DRSA com o objetivo de apoiar decisões quanto à destinação de recursos para o combate às ações de roubos.
\end{abstract}

Palavra-chave: Segurança pública; Multicritério; Roubos.

\begin{abstract}
The decision model application in the context of public security is interesting because of the capacity to support the decision-maker choices through the use of the mathematical approach. Given the monetary and people restrictions, the actions to resources allocation need to satisfy the limitations and, at the same time, propose good solutions. That so, the classification of the level of vulnerability can be considered to minimize the crime occurrences. In this way, the DRSA methodology was applied aiming to support the resources allocation in public security.
\end{abstract}

Keywords: Public security; Multicriteria; Robberies.

\section{Como Citar:}

ROSA, A. G. F.; MOTA, C. M. M.; FIGUEIREDO, C. J. J.. Classificação de vulnerabilidade de áreas à roubos. In: SIMPÓSIO DE PESQUISA OPERACIONAL E LOGÍSTICA DA MARINHA, 19., 2019, Rio de Janeiro, RJ. Anais [...]. Rio de Janeiro: Centro de Análises de Sistemas Navais, 2019. 


\section{INTRODUÇÃO}

Tendo em vista a importância da avaliação do contexto social, demográfico e espacial na dinâmica de ocorrência dos crimes de roubo em vias públicas, e seu relacionamento com os fluxos de moedas, pessoas e mercadorias [1] [2] [3] [4] [5] 6] [7, o presente artigo apresenta o processo de construção do modelo multicritério para o suporte de ações de alocação de recursos para o combate aos roubos.

Sobre a construção do modelo de decisão, tem-se o emprego do DRSA (Dominancebased Rough Set Approach) devido a sua capacidade de levar o decisor a um aprendizado acerca das suas preferências a partir da avaliação da qualidade e do conjunto de regras de decisão gerado da análise de um subconjunto de alternativas, podendo ser estendido para todo o conjunto de amostra levantada [8] 9 .

O DRSA é um método que possui origem na teoria dos conjuntos rough, que consiste basicamente na agregação de elementos similares numa mesma classe; e sendo indiscerníveis, os objetos pertencentes a uma mesma classe seriam chamados de elementares. Contudo, o que acontece na realidade é que esses objetos possuem alguns aspectos que os diferem dos demais. Assim, a teoria dos conjuntos rough em si não é suficiente para a avaliação de objetos que apresentam características de dominância em relação a outros. Dessa forma, surge o DRSA, que diferente da visão tradicional, traz em sua formulação o princípio da dominância, utilizado no presente estudo por se considerar a classificação dos elementos em níveis ordenados de vulnerabilidade [10] [11] [12].

Assim, tem-se que o objetivo do estudo é construir uma ferramenta de decisão que possa classificar áreas em grupos de vulnerabilidade para o direcionamento da alocação de recursos, seja de efetivo, patrulhas ou investimento em equipamentos.

Nesse sentido, buscou-se desenvolver o modelo de decisão para uma localidade do estado de Pernambuco, cujo o nome não será divulgado, tendo em vista o compromisso ético da pesquisa em não ferir, expor ou estereotipar as pessoas desses locais, e nem causar quaisquer tipos de prejuízos econômico e social para a área avaliada no estudo.

O trabalho está dividido em 6 seções. A segunda seção trata da apresentação da metodologia que descreve as etapas de realização do trabalho. Na terceira seção, tem-se os resultados. A quarta seção apresenta algumas conclusões, na quinta tem-se os agradecimentos e na sexta as referências.

\section{METODOLOGIA}

Conforme levantado anteriormente, a dinâmica dos crimes está associada aos fluxos de dinheiro, pessoas e mercadorias em um dado espaço. Assim, avaliar esses aspectos na construção do modelo de decisão para o propósito de aplicação na área de segurança pública torna-se interessante.

Inicialmente, o estudo consistiu em buscas bibliográficas com o propósito de fundamentação e coleta de informações razoáveis para a construção do modelo, conforme alguns estudos realizados na área. Em seguida, buscou-se estabelecer relações entre as variáveis levantadas com as ocorrências de roubos.

As variáveis levantadas consistiram em dados do Censo 2010, coletados na base 
do IBGE (Instituto Brasileiro de Geografia e Estatística) [13, e localização espacial das paradas de ônibus coletadas diretamente no Open Street Maps (OSM).

Algumas considerações foram feitas quanto as análises para relação das variáveis com os crimes de roubos. Os dados extraídos do IBGE em conjunto com o número de paradas de ônibus distribuídas na área estudada foram submetidos ao cálculo de binomial negativa, devido a dispersão dos dados [14, para fins exploratórios, Tabela 1.

Tabela 1: Binomial negativa sobre os dados do Censo 2010

\begin{tabular}{|l|l|}
\hline Variáveis explicativas & P-valor \\
\hline $\mathbf{n}^{\mathbf{o}}$ de domicílios & 0,7594 \\
\hline $\mathbf{n}^{\mathbf{o}}$ de domicílios abastecidos com água & 0,61402 \\
\hline $\mathbf{n}^{\mathbf{o}}$ de domicílios com coleta de lixo & 0,53574 \\
\hline $\mathbf{n}^{\mathbf{o}}$ de domicílios com energia & 0,33705 \\
\hline moradia em condições improvisadas & 0,17743 \\
\hline renda total & 0,58766 \\
\hline $\mathbf{n}^{\mathbf{o}}$ de alfabetizados & 0,13625 \\
\hline total de residentes & $0,00949^{* *}$ \\
\hline $\mathbf{n}^{\mathbf{o}}$ de paradas de ônibus & $2 e^{-16} * * *$ \\
\hline
\end{tabular}

Fonte: Elaboração própria (2019).

Conforme a Tabela 1, o total de residentes e o número de paradas de ônibus se apresentam como mais significativos na análise de roubos (note-se pela obtenção do p-valor pequeno, que confere a essas duas variáveis mais de $99 \%$ de significância).

\subsection{Construção do modelo}

A partir da revisão da literatura e da exploração estatística, foi possível realizar o levantamento de indicadores capazes de exprimir desigualdade, fluxos e concentração de pessoas, fatores de decisão atrelados a propensão da ocorrência do roubo. Assim, o número de domicílios improvisados, quantidade total de residentes, número de alfabetizados e distribuição de paradas de ônibus puderam ser considerados como critérios de avaliação das alternativas [5] [4] [15] [16] [17] [6] [18] [19].

Assim, a análise do modelo que visa o entendimento do problema abordado e objetiva a verificação da viabilidade do seu uso na ordenação das alternavas em níveis de vulnerabilidade para o apoio na alocação de recursos pode ser realizada. Dessa forma, o estudo segue as etapas metodológicas apresentadas na Figura 1. 
Figura 1: Construção do modelo

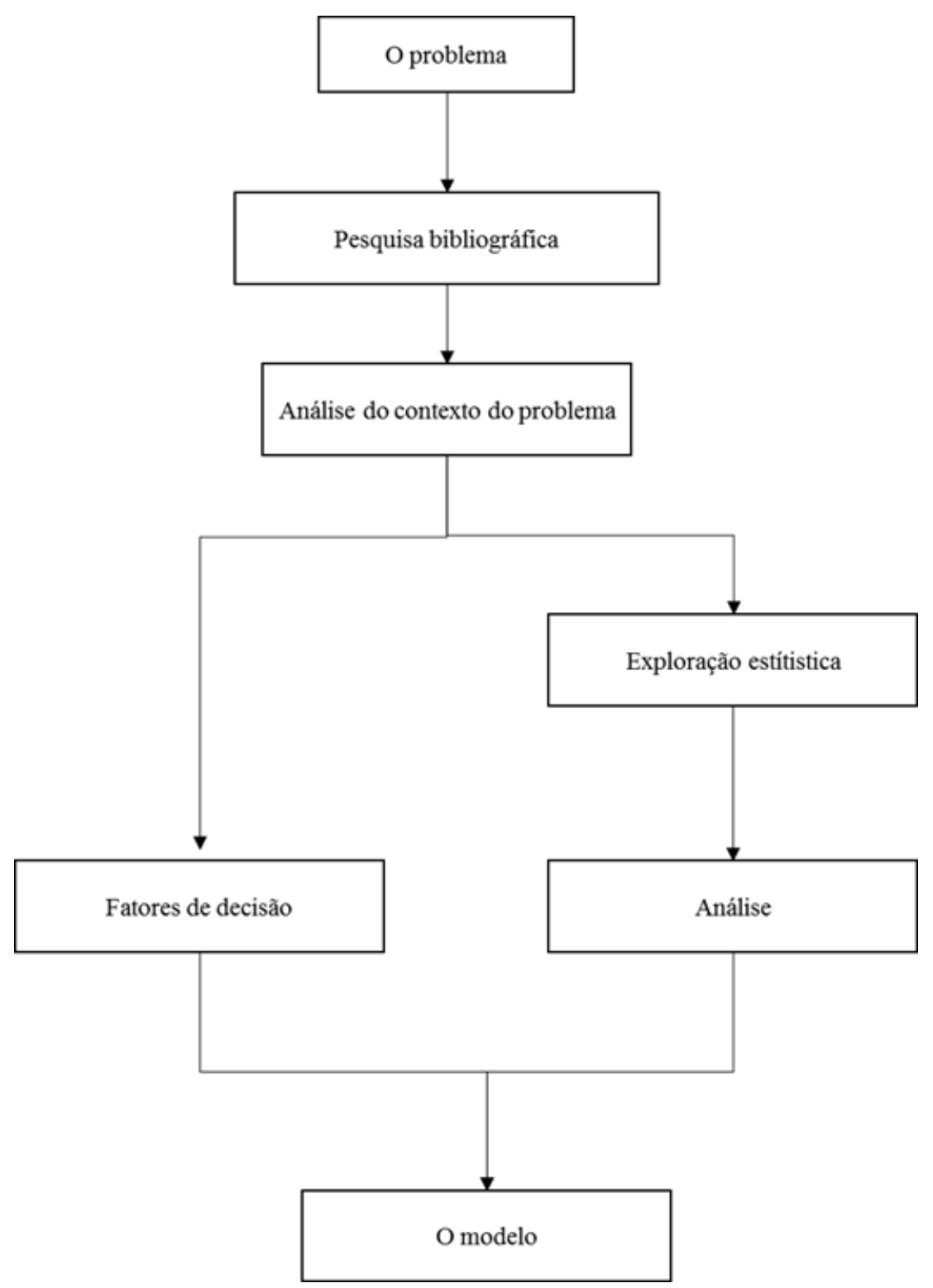

Fonte: Elaboração própria (2019).

Conforme a Figura 1, a construção do modelo parte da identificação do problema, sendo realizadas pesquisas sobre a temática e posterior análise do contexto que envolve esse evento, se ramificando em observação dos fatores que implicam na tomada de decisão e exploração dos dados de roubos e dos elementos demográficos, para obtenção do modelo.

Conforme discutido, o estudo opta pelo uso do DRSA porque considera os dados a priori, além de promover a reflexão do problema por meio das regras de decisão e classificar, ordenadamente, as alternativas em níveis de vulnerabilidade.

\section{RESULTADOS}


Estabelecido o ambiente de estudo e selecionados os critérios obtidos do Censo 2010 e de localização das paradas de ônibus (considerada no estudo um elemento proxy de descrição de fluxos), verificou-se o comportamento do modelo para um subconjunto de 18 alternativas (setores censitários) extraídas do total de 155 para a geração do conjunto de regras de decisão, conforme a Tabela 2 .

Tabela 2: Regras de decisão para dados do Censo 2010 e paradas

\begin{tabular}{|c|l|l|}
\hline Regra & Decisão & Condições \\
\hline 1 & preferência $>=$ vulnerabilidade muito alta & paradas $>=81$ \\
2 & preferência $>=$ vulnerabilidade muito alta & alfabetizados $>=833$ \\
3 & preferência $>=$ vulnerabilidade alta & paradas $>=9$ \\
4 & preferência $>=$ vulnerabilidade média & alfabetizados $>=487$ \\
5 & preferência $>=$ pouco vulnerável & alfabetizados $>=444$ \\
6 & preferência $>=$ pouco vulnerável & domicílio improvisado preferência $>1$ \\
7 & preferência $>=$ pouco vulnerável & alfabetizados $>=191 ;$ total residentes $<=275$ \\
8 & preferência $<=$ nada vulnerável & alfabetizados $<=0$ \\
9 & preferência $<=$ nada vulnerável & alfabetizados $<=400$ \\
10 & preferência $<=$ pouco vulnerável & alfabetizados $>=452 ;$ total residentes $>=443$ \\
11 & preferência $<=$ vulnerabilidade média & alfabetizados $<452 ;$ paradas $<=2$ \\
12 & preferência $<=$ vulnerabilidade alta & paradas $<=4$ \\
13 & preferência $<=$ vulnerabilidade alta & alfabetizados $<=250$ \\
14 & preferência $<=$ vulnerabilidade alta & total residentes $>=975$ \\
\hline
\end{tabular}

Fonte: Elaboração própria (2019).

As regras de decisão geradas para as 18 alternativas representantes dos diferentes níveis de ameaça de roubo, puderam ser estendidas aos outros setores. Sendo possível a criação do mapa de apontamento de vulnerabilidade, onde os setores censitários se agrupam em cinco níveis de risco: nada vulnerável, pouco vulnerável, vulnerabilidade média, vulnerabilidade alta e vulnerabilidade muito alta, conforme o apresentado na Figura 2 .

Figura 2: Avaliação de vulnerabilidade (dados do Censo 2010 e de paradas de ônibus)

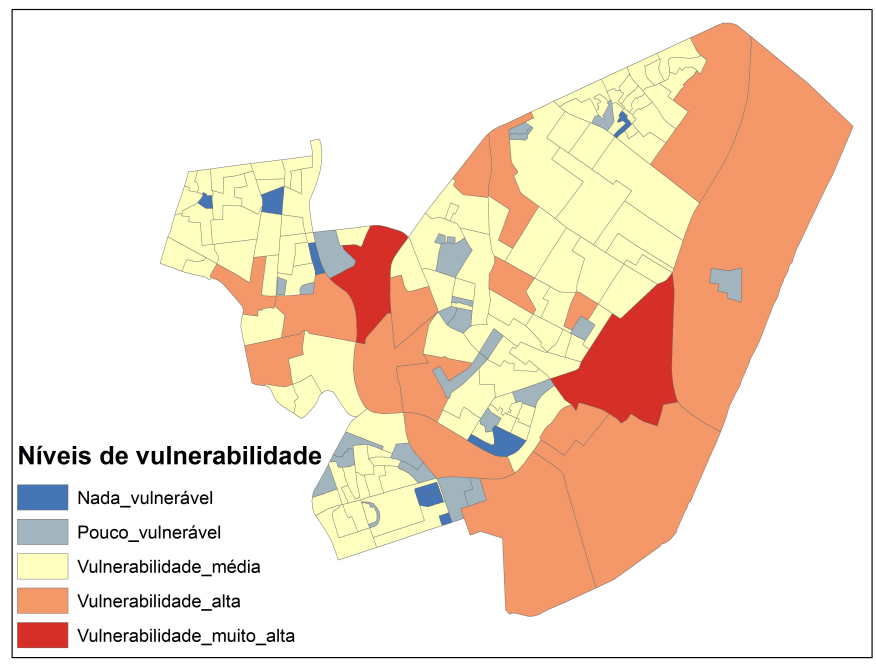

Fonte: Elaboração própria (2019). 
Segundo o que se verifica na Figura 2, dois setores se classificam como de vulnerabilidade muito alta, enquanto um número maior de setores censitários de tamanho relativamente pequeno, apresentam vulnerabilidade média. Além disso, pode-se verificar uma correspondência espacial de risco, locais de vulnerabilidade muito alta estão próximos a locais de vulnerabilidade alta e os setores se tornam menos perigosos à medida que se afastam dos locais mais críticos.

\section{CONCLUSÕES}

Como se verifica no presente trabalho, a construção do modelo de decisão deve abordar diferentes perspectivas a partir da avaliação holística do problema. De tal forma que se consiga apoiar ações que surtam o efeito de combate aos crimes de roubos em vias públicas.

Sob esse ponto de vista, acrescenta-se que o estudo se mostra útil na avaliação da propensão do modelo de decisão como ferramenta de apoio para a alocação de recurso para o setor público, em especial ao planejamento na área de segurança pública, temática abordada no presente trabalho.

Considera-se, portanto, que o trabalho reforça a aplicação de uma ferramenta fundamentada matematicamente que leva em consideração o levantamento e exploração de dados reais. Nesse sentido, para pesquisas futuros cogita-se aperfeiçoamentos no modelo desenvolvido da mesma maneira que se busca realizar o estudo visando a coleta de parâmetros de eficiência do modelo para seja continuamente melhorado

\section{AGRADECIMENTOS}

Os autores agradecem o apoio financeiro da CAPES.

\section{REFERÊNCIAS BIBLIOGRÁFICAS}

[1] BRANTINGHAM, P. L.; BRANTINGHAM, P. J. Nodes, paths and edges: considerations on the complexity of crime and the physical environment. Journal of Environmental Psychology, v. 13, n. 1, p. 3-28, 1993. 2

[2] OSGOOD, D. W.; CHAMBERS, J. M. SOCIAL DISORGANIZATION OUTSIDE THE METROPOLIS: AN ANALYSIS OF RURAL YOUTH VIOLENCE*. Criminology, v. 38, n. 1, p. 81-116, 2 2000. ISSN 0011-1384. Disponível em: <http://doi.wiley.com/10.1111/j.1745-9125.2000.tb00884.x>. 2

[3] YARBROUGH, A. et al. Social Learning and Self-Control: Assessing the Moderating Potential of Criminal Propensity. International Journal of Offender Therapy and Comparative Criminology, v. 56, n. 2, p. 191-202, 4 2012. ISSN 0306-624X. Disponível em: <http://journals.sagepub.com/doi/10.1177/0306624X10396041>. 2

[4] NEWTON, A. D.; PARTRIDGE, H.; GILL, A. Above and below: measuring crime risk in and around underground mass transit systems. Crime Science, v. 3, n. 1, 2014. ISSN 2193-7680. Disponível em: 
$<$ http://crimesciencejournal.springeropen.com/articles/10.1186/2193-7680-3-1>.

2, 3

[5] YU, S.-s. V.; MAXFIELD, M. G. Ordinary Business: Impacts on Commercial and Residential Burglary. British Journal of Criminology, v. 54, n. 2, p. 298320, 3 2014. ISSN 0007-0955. Disponível em: <https://academic.oup.com/bjc/articlelookup/doi/10.1093/bjc/azt064>. 2, 3

[6] WEISBURD, D.; GROFF, E. R.; YANG, S.-M. The Importance of Both Opportunity and Social Disorganization Theory in a Future Research Agenda to Advance Criminological Theory and Crime Prevention at Places. Journal of Research in Crime and Delinquency, v. 51, n. 4, p. 499-508, 7 2014. ISSN 0022-4278. Disponível em: <http://journals.sagepub.com/doi/10.1177/0022427814530404>. 2 , 3

[7] GERELL, M. Bus Stops and Violence, Are Risky Places Really Risky? European Journal on Criminal Policy and Research, 4 2018. ISSN 0928-1371. Disponível em: <http://link.springer.com/10.1007/s10610-018-9382-5>. 2

[8] SLOWINSKI, R.; GRECO, S.; MATARAZZO, B. Rough set and rule-based multicriteria decision aiding. Pesquisa Operacional, v. 32, n. 2, p. 213-270, 8 2012. ISSN 0101-7438. Disponível em: <http://www.scielo.br/scielo.php?script $=\mathrm{sci}_{a}$ rttextpid $=$ $S 0101-74382012000200001$ lng $=$ entlng $=$ en:.2

[9] KADZIŃSKI, M.; GRECO, S.; SŁOWIŃSKI, R. Robust Ordinal Regression for Dominance-based Rough Set Approach to multiple criteria sorting. Information Sciences, v. 283, p. 211-228, 2014. ISSN 00200255. 2

[10] PAWLAK, Z. Rough set approach to knowledge-based decision support. European Journal of Operational Research, v. 99, n. 1, p. 48-57, 1997. ISSN 03772217. Disponível em: <http://www.sciencedirect.com/science/article/pii/S0377221796003827>. 2

[11] PAWLAK, Z. Rough sets: Some extensions. Information Sciences, Elsevier, v. $177, \quad$ n. 1 , p. 28-40, 1 2007. ISSN 0020-0255. Disponível em: <https://www.sciencedirect.com/science/article/pii/S0020025506001496>. 2

[12] GRECO, S.; SŁOWIŃSKI, R.; ZIELNIEWICZ, P. Putting Dominance-based Rough Set Approach and robust ordinal regression together. Decision Support Systems, v. 54, n. 2, p. 891-903, 2013. ISSN 01679236. 2

[13] IBGE. Conteúdo do diretório. 2016. Disponível em: <ftp://ftp.ibge.gov.br/Censos/Censo_Demografico_2010>. 3

[14] HOEF, J. M. V.; BOVENG, P. L. Quasi-Poisson vs. Negative Binomial Regression: how should we model overdispersed count data? Ecology, v. 88, n. 11, p. 2766-2772, 11 2007. ISSN 0012-9658. Disponível em: <http://doi.wiley.com/10.1890/07-0043.1>. 3

[15] BRANTINGHAM, P. J.; BRANTINGHAM, P. L. Environmental criminology: from theory to urban planning practice. Studies on crime and crime prevention, v. 7, n. 1 , p. $31-60,1998.3$

[16] LEVINE, N.; WACHS, M. Bus crime in Los Angeles: I - Measuring the incidence. Transportation Research Part A: General, v. 20, n. 4, p. 273-284, 1986. 3 
[17] LIMA, F. S. de; MARINHO, E. Public security in Brazil: Efficiency and technological gaps. EconomiA, Elsevier, v. 18, n. 1, p. 129-145, 1 2017. ISSN 1517-7580. Disponível em: <https://www.sciencedirect.com/science/article/pii/S1517758016301072>. 3

[18] FIGUEIREDO, C. J. J. d.; MOTA, C. M. d. M. A Classification Model to Evaluate the Security Level in a City Based on GIS-MCDA. Mathematical Problems in Engineering, p. 1-10, 2016. ISSN 1024-123X. Disponível em: <http://www.hindawi.com/journals/mpe/2016/3534824/>. 3

[19] PEREIRA, D. V. S.; MOTA, C. M. M.; AndRESEN, M. A. The homicide Drop in Recife, Brazil: A study of Crime Concentrations and Spatial Patterns. Homicide Studies, v. 21, n. 1, p. 21-38, 2017. 3 